\title{
Chaotic scattering of atoms at a standing laser wave
}

\author{
S. V. Prants
}

Laboratory of Nonlinear Dynamical Systems, Pacific Oceanological Institute of the Russian Academy of Sciences, 43 Baltiiskaya st., 690041 Vladivostok, Russia, URL: dynalab.poi.dvo.ru

PACS 05.45.Mt - Quantum chaos; semiclassical methods

PACS $37.10 . \mathrm{Vz}$ - Mechanical effects of light on atoms, molecules, and ions

PACS 42.50.St - Nonclassical interferometry, subwavelength lithography

\begin{abstract}
Atoms, propagating across a detuned standing laser wave, can be scattered in a chaotic way even in the absence of spontaneous emission and any modulation of the laser field. Spontaneous emission masks the effect in some degree, but the Monte Carlo simulation shows that it can be observed in real experiments by the absorption imaging method or depositing atoms on a substrate. The effect of chaotic scattering is explained by a specific behavior of the dipole moments of atoms crossing the field nodes and is shown to depend strongly on the value of the atom-laser detuning.
\end{abstract}

Introduction. - The deflection of an atomic beam it a laser standing wave (SW) is explained by the dipole forces which are well described by the classical atom-field interaction model [1,2. The ability of a SW to diffract, focus or splitting an atomic beam 3 has been used for à variety of applications including atom microscopy, interferometry, isotope separation, and optical lithography [4] 6]. On the other hand, cold atoms are ideal candidates to test fundamental principles of quantum physics includ.ing the phenomenon of dynamical chaos at the microscopic level that is known as a kind of random-like motion in à deterministic environment $7-16$. Dynamical chaos is characterized by exponential sensitivity of trajectories in - the phase space to small variations in initial conditions and/or control parameters.

It has been proposed in Ref. [7] to study quantum chaos and the corresponding effect of dynamical localization placing cold atoms in a far-detuned SW with a periodic kick-like modulation of positions of the SW nodes. A number of experiments 9 have been carried out in accordance with this proposal. At large detunings, the atoms are not excited being quantum analogues of classical kick rotors. Since those experiments on atom optics realization of the $\delta$-kicked quantum rotor, cold atoms provide new grounds for experiments and theory on quantum chaos.

It has been shown in Ref. [10 that even a single-pulse far-detuned SW can induce chaos in atomic motion. For sufficiently large detuning, the excited state amplitude can be adiabatically eliminated [7, leading to a Hamiltonian with an external degree of freedom only. The corresponding equations of motion for an externally modulated nonlinear pendulum constitute the well-known model with one and half degree of freedom that can be chaotic under some conditions. The other possibility is to induce chaos in spin degree of freedom of atoms periodically kicked by applying short magnetic field pulses [11. That is the one and half degree of freedom model of a kicked top.

In difference from those and other papers on the related topic, we consider the physical situation with comparatively small detunings and should take into account a coupling between external and internal atomic dynamics, leading to a model with three degrees of freedom. It will be shown in the present paper that in this case one needs no modulation or any other perturbation of the SW to induce chaotic internal and external dynamics of atoms crossing the SW laser field.

Near the atom-field resonance, when the interaction between the internal and external atomic degrees of freedom is intense, there is a possibility to create conditions for chaotic behavior without any kicking and modulation [15 17. If so, it is open the way to test the novel regime of atomic motion caused by the peculiarities of the dipole force in the strong coupling regime. In the semiclassical approximation, atom with quantized internal dynamics is treated as a point-like particle with the HamiltonSchrödinger equations of motion constituting a nonlinear 
dynamical system [15]18. In a certain range of the atomfield detunings, a set of atomic trajectories becomes exponentially sensitive to small variations in initial quantum internal and classical external states or/and in the control parameters. Hamiltonian evolution is a smooth process that is well described in a semiclassical approximation by the Hamilton-Schrödinger equations. The problem becomes much more complicated because of spontaneous emission of atoms with a specific shot quantum noise acting in a dynamical system which is chaotic in the absence of noise. A number of nonlinear Hamiltonian and dissipative effects have been found numerically and analytically near the resonance including chaotic Rabi oscillations, chaotic atomic transport, dynamical fractals, and Lévy flights 15,22 .

The main aim of the paper is to demonstrate theoretically and numerically that the new type of atomic diffraction at a rigid SW without any modulation, chaotic atomic scattering, can be observed in a real experiment. The scheme of such an experiment is shown in Fig. 1 with a beam of atoms crossing a SW laser field. One either measures a spatial atomic distribution after the interaction by the absorption imaging technique or measures an atomic distribution on a silicon substrate in the far-field zone. The results are expected to be different depending on the value of the atom-field detuning. The distribution is expected to be comparatively narrow at those values of the detuning at which atomic scattering is regular (r.s. distribution in Fig. 1) or wide at the detuning values providing chaotic scattering of atoms due to their chaotic walking along the SW (c.s. distribution in Fig. 1).

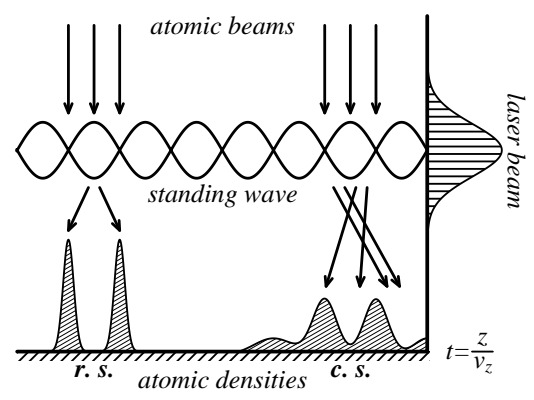

Fig. 1: Schematic representation of the proposed experiment on regular (r.s.) and chaotic (c.s.) atomic scattering at a Gaussian standing laser wave.

\section{Results. -}

Main equations and the regimes of atomic motion. A beam of two-level atoms in the $z$ direction crosses a SW laser field with optical axis in the $x$ direction (Fig.11). The laser field amplitude has the Gaussian profile $\exp \left[-\left(z-z_{0}\right)^{2} / r^{2}\right]$ with $r$ being the $e^{-2}$ radius at the laser beam waist. The characteristic length of the atom-field interaction is supposed to be $3 r$ because the light intensity at $z=z_{0} \pm 1.5 r$ is two orders of magnitude smaller than the peak value. The longitudinal velocity of atoms, $v_{z}$, is much larger than their transversal velocity $v_{x}$ and is supposed to be constant. Thus, the spatial laser profile may be replaced by the temporal one. The Hamiltonian of an atom in the 1D SW field can be written in the frame rotating with the laser frequency $\omega_{f}$ as follows:

$$
\hat{H}=\frac{P^{2}}{2 m_{a}}+\frac{\hbar}{2}\left(\omega_{a}-\omega_{f}\right) \hat{\sigma}_{z}-
$$

$\hbar \Omega_{0} \exp \left[-\left(t-\frac{3}{2} \sigma_{t}\right)^{2} / \sigma_{t}^{2}\right]\left(\hat{\sigma}_{-}+\hat{\sigma}_{+}\right) \cos k_{f} X-\frac{i \hbar \Gamma}{2} \hat{\sigma}_{+} \hat{\sigma}_{-}$,

where $\hat{\sigma}_{ \pm, z}$ are the Pauli operators for the internal atomic degrees of freedom, $X$ and $P$ are the classical atomic position and momentum, $\Gamma, \omega_{a}$, and $\Omega_{0}$ are the decay rate, the atomic transition and maximal Rabi frequencies, respectively, and $\sigma_{t} \equiv r / v_{z}$, i.e., $3 \sigma_{t}$ is the transit time. The wavefunction for the electronic degree of freedom is $|\Psi(t)\rangle=a(t)|2\rangle+b(t)|1\rangle$, where $a \equiv A+i \alpha$ and $b \equiv B+i \beta$ are the probability amplitudes to find the atom in the excited, $|2\rangle$, and ground, $|1\rangle$, states, respectively.

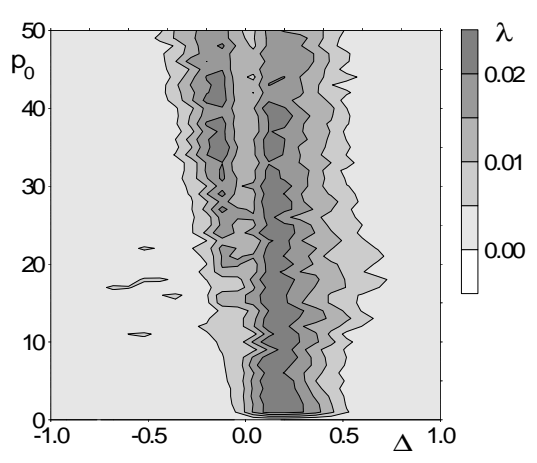

Fig. 2: Finite-time Lyapunov exponent $\lambda$ vs atom-field detuning $\Delta$ (in units of the Rabi frequency $\Omega_{0}$ ) and initial atomic transversal momentum $p_{0}$ (in units of the photon momentum $\left.\hbar k_{f}\right)$ at the normalized recoil frequency $\omega_{r}=10^{-3}$ and $\gamma=0$.

In the semiclassical approximation, atom with quantized internal dynamics is treated as a point-like particle (the transversal atomic momentum $p$ is supposed to be, in average, much larger than the photon one, $\hbar k_{f}$ ) with the equations of motion written for the real and imaginary parts of the probability amplitudes

$$
\begin{gathered}
\dot{x}=\omega_{r} p, \dot{p}=-2 \exp \left[-\left(\tau-\frac{3}{2} \sigma_{\tau}\right)^{2} / \sigma_{\tau}^{2}\right](A B+\alpha \beta) \sin x \\
\dot{A}=\frac{1}{2}\left(\omega_{r} p^{2}-\Delta\right) \alpha-\frac{1}{2} \gamma A-\exp \left[-\left(\tau-\frac{3}{2} \sigma_{\tau}\right)^{2} / \sigma_{\tau}^{2}\right] \beta \cos x \\
\dot{\alpha}=-\frac{1}{2}\left(\omega_{r} p^{2}-\Delta\right) A-\frac{1}{2} \gamma \alpha+\exp \left[-\left(\tau-\frac{3}{2} \sigma_{\tau}\right)^{2} / \sigma_{\tau}^{2}\right] B \cos x \\
\dot{B}=\frac{1}{2}\left(\omega_{r} p^{2}+\Delta\right) \beta-\exp \left[-\left(\tau-\frac{3}{2} \sigma_{\tau}\right)^{2} / \sigma_{\tau}^{2}\right] \alpha \cos x \\
\dot{\beta}=-\frac{1}{2}\left(\omega_{r} p^{2}+\Delta\right) B+\exp \left[-\left(\tau-\frac{3}{2} \sigma_{\tau}\right)^{2} / \sigma_{\tau}^{2}\right] A \cos x
\end{gathered}
$$



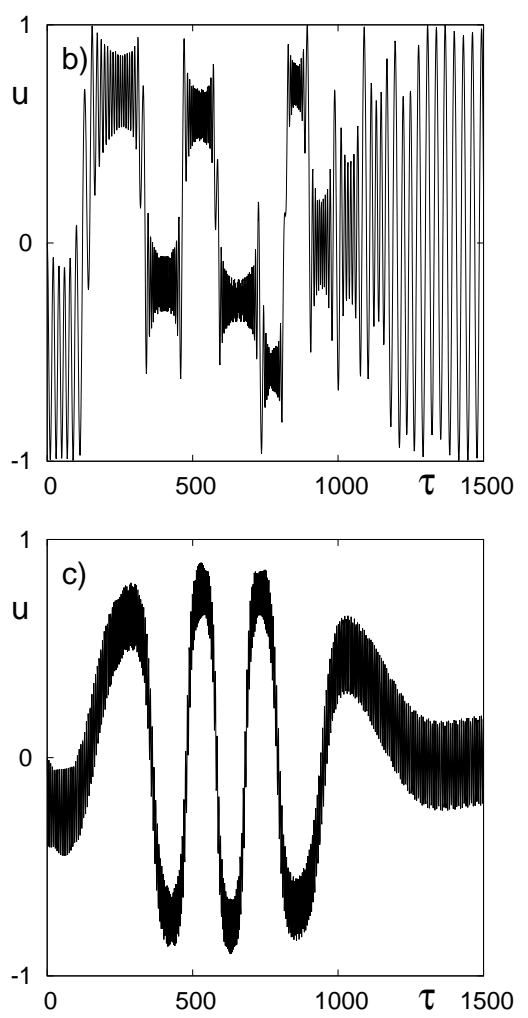

Fig. 3: Evolution ( $\tau$ is in units of $\Omega_{0}^{-1}$ ) of the atomic dipolemoment component $u=2(A B+\alpha \beta)$ in (a) the chaotic $(\Delta=$ $0.2)$ and $(b)$ regular $(\Delta=1)$ regimes of atomic motion.

where $x \equiv k_{f} X$ and $p \equiv P / \hbar k_{f}$ are atomic center-of-mass position and transversal momentum, respectively and dot denotes differentiation with respect to the dimensionless time $\tau \equiv \Omega_{0} t$. The recoil frequency, $\omega_{r} \equiv \hbar k_{f}^{2} / m_{a} \Omega_{0} \ll 1$, the atom-laser detuning, $\Delta \equiv\left(\omega_{f}-\omega_{a}\right) / \Omega_{0}$, the decay rate $\gamma=\Gamma / \Omega_{0}$, and the characteristic interaction time, $\sigma_{\tau} \equiv r \Omega_{0} / v_{z}$, are the control parameters.

Without the decay, $\gamma=0$, Eqs. (2) constitute the nonlinear Hamiltonian dynamical system with three degrees of freedom describing an atom moving in the six-dimensional phase space. The simple way to know how complicated this motion may be is to compute the quantitative measure of chaos, maximal Lyapunov exponent characterizing the mean rate of the exponential divergence of initially close trajectories. Because of a transient character of chaos we compute the finite-time Lyapunov exponent $\lambda$, i.e. the value of the exponent at the moment when atoms leave the interaction region. The result of computation with Eqs. (2) at the given value of the recoil frequency, $\omega_{r}=10^{-3}$, and zero decay rate in dependence on the detuning $\Delta$ and the initial atomic transversal momentum $p_{0}$ is shown in Fig. 2. Color codes the magnitude of the finitetime Lyapunov exponent. In white regions the values of $\lambda$ are almost zero, and the internal and translational motion is regular in the corresponding ranges of $\Delta$ and $p_{0}$. In shadowed regions positive values of $\lambda$ imply unstable
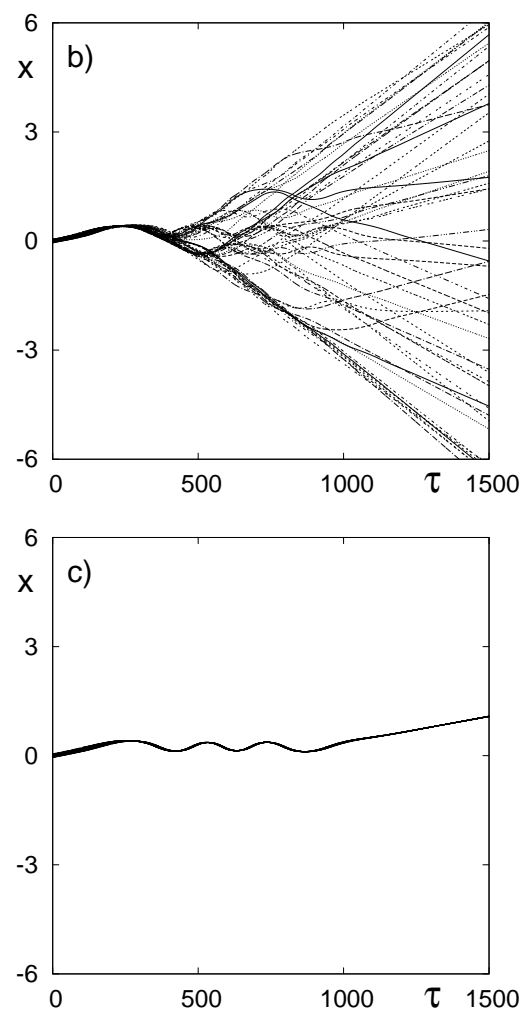

Fig. 4: Trajectories in the real space for 50 atoms without spontaneous emission. Hamiltonian (a) chaotic $(\Delta=0.2)$ and (b) regular scattering $(\Delta=1)$. The atomic position $x$ is in units of the optical wavelength.

motion.

The scheme of the proposed experiment in Fig. 11 resembles the scattering problem with particles entering an interaction region along completely regular trajectories and leaving it along asymptotically regular trajectories 2328 . However, in difference from the standard chaotic scattering with a nonattractive fractal invariant set existing over an infinite time, this process may be interpreted as a transient chaos or a finite-time chaotic scattering.

There are three possible chaotic regimes of the center-ofmass motion along the SW optical axis [17,18. In dependence on the initial conditions and the values of the control parameters, atoms may oscillate chaotically in wells of the optical potential or move ballistically over its hills with chaotic variations of their velocity. Chaotic motion becomes possible in a narrow range of the detuning values, $0<|\Delta|<1$. At $\Delta=0$, the synchronized electric-dipole component, $u=2(A B+\alpha \beta)$ becomes a constant. That implies the additional integral of motion in the Hamiltonian version of Eqs.(2) and the regular motion with $\lambda=0$. Far from the resonance, at $|\Delta|>1$, the motion is again regular both in the trapping and flight modes. That speculation is confirmed by the Lyapunov map in Fig. 2 ,

Moreover, there is a specific type of motion, chaotic walking in a deterministic optical potential, when atoms 

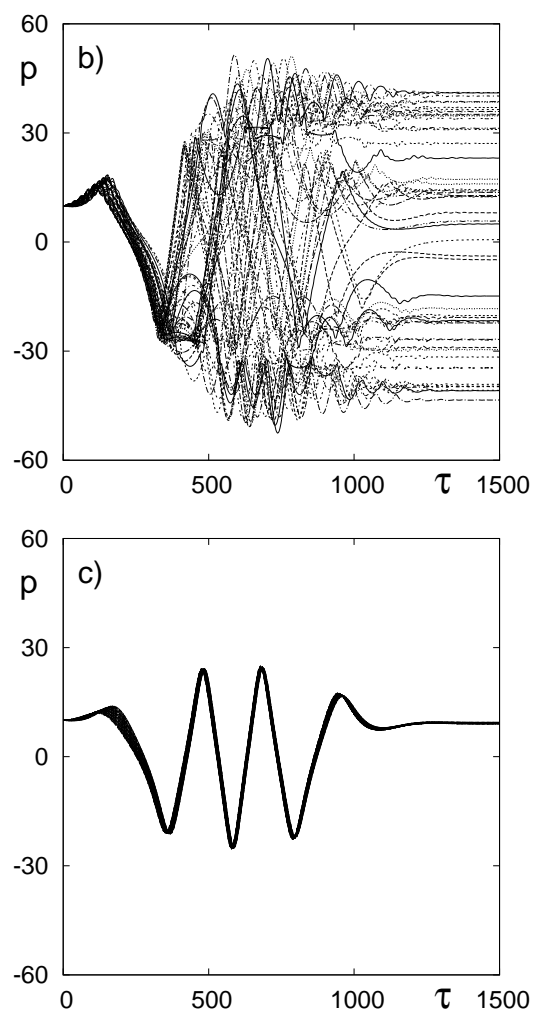

Fig. 5: The same as Fig. 4 but in the momentum space.

can change the direction of motion alternating between flying through the SW and being trapped in its potential wells. We would like to stress that the local instability produces chaotic center-of-mass motion in a rigid SW without any modulation of its parameters in difference from the case with periodically kicked and far detuned optical lattices [7, 9 11. The trivial time dependence in the Hamiltonian (2) cannot produce chaotic motion, it simply accounts for a modulation of the interaction of atoms with a Gaussian laser beam. Even if the atoms would cross an absolutely homogeneous (in the $z$ direction) laser beam there would be under appropriate conditions chaotic atomic center-of-mass motion in the transversal $x$-direction.

Chaotic walking occurs due to the specific behavior of the Bloch-vector component, $u$, of a moving atom whose shallow oscillations between the SW nodes are interrupted by sudden jumps with different amplitudes while atom crosses each node [18. We illustrate in Fig. 3 the behavior of the $u$ component with different values of the detuning $\Delta=0.2$ and $\Delta=1$ at which the atomic motion in accordance with the $\lambda$-map in Fig. 2 is chaotic and regular, respectively. The time of the atomic interaction with the SW field is estimated to be $3 \sigma_{\tau}=1200$. So, the jumps of the $u$ variable (if any) disappear after that time in Fig. 3 . It follows from the second equation in the set (2) that jumps in the variable $u=2(A B+\alpha \beta)$ result in jumps of the atomic momentum while crossing a node of the SW. If the value of the atomic energy is close to a separatrix one, the atom after the corresponding jump-like change in $p$ can either overcome the potential barrier and leave a potential well or it will be trapped by the well, or it will move as before. The jump-like behavior of $u$ is the ultimate reason of chaotic atomic walking along a deterministic SW.

It is easy to estimate the range of initial momenta at which atoms are expected to change their direction of motion or move ballistically. At small detunings, $|\Delta| \ll 1$, the total energy consists of the kinetic one, $K=\omega_{r} p^{2} / 2$, and the potential one, $U=u \cos x$. If $K(\tau=0)>\left|U_{\max }\right|=1$, then the atom will move ballistically. This occurs if the initial atomic momentum, $p_{0}$, satisfies to the condition $p_{0}>\sqrt{2 / \omega_{r}}>44$. If the initial conditions are chosen to give $0 \leq K(\tau=0)+U(\tau=0) \leq 1$, the atoms with $0 \lesssim p_{0} \lesssim 44$ are expected to perform a chaotic walking at positive $\Delta$.
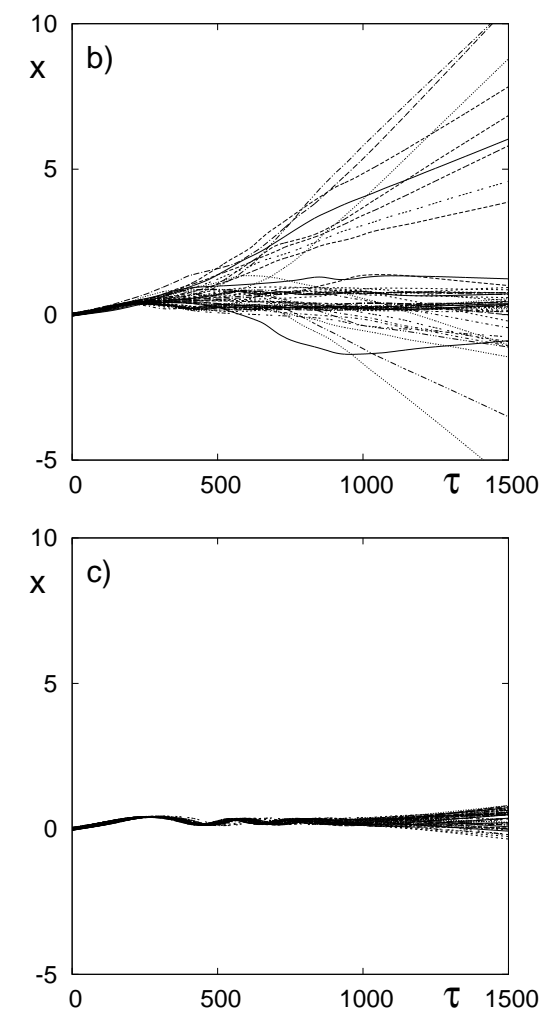

Fig. 6: Scattering of 50 spontaneously emitting atoms at the SW with the decay rate $\gamma=0.05$ and the same other conditions as in Fig. 4 (a) Chaotic $(\Delta=0.2)$ and (b) regular $(\Delta=1)$ regimes.

Hamiltonian chaotic scattering. $\quad$ Let us consider a spatially uniform and previously focused beam of atoms crossing a Gaussiam laser beam. The position and momentum distributions of atoms are measured after interaction with the SW field. We predict that those distributions would be much broader at those values of $\Delta$ at which one expects chaotic walking to occur. Firstly, we perform simulation with a negligible probability of sponta- 

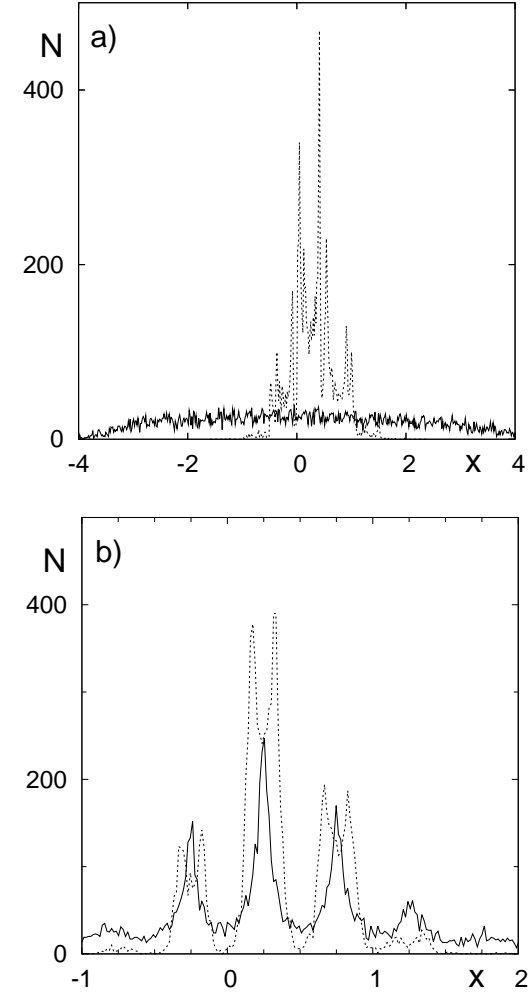

Fig. 7: The distributions of $10^{4}$ lithium atoms (a) without and (b) with spontaneous emission at $\tau=1000$ under the conditions of chaotic scattering at $\Delta=0.2$ (bold curves) and regular scattering at $\Delta=1$ (dashed curves).

neous emission. To be concrete let us take calcium atoms with the working intercombination transition $4^{1} S_{0}-4^{3} P_{1}$ at $\lambda_{a}=657.5 \mathrm{~nm}$, the recoil frequency $\nu_{\mathrm{rec}} \simeq 10 \mathrm{KHz}$, and the lifetime of the excited state $T_{\mathrm{sp}}=0.4 \mathrm{~ms}$. Taking the maximal Rabi frequency to be $\Omega_{0} / 2 \pi=2 \cdot 10^{7} \mathrm{~Hz}$, the radius of the laser beam $r=0.3 \mathrm{~cm}$, and the mean longitudinal velocity $v_{z}=10^{3} \mathrm{~m} / \mathrm{s}$, the interaction time is estimated to be $0.9 \mathrm{~ms}$. The normalized recoil frequency is $\omega_{r}=4 \pi \nu_{\mathrm{rec}} / \Omega_{0}=10^{-3}$ and $\sigma_{\tau}=400$.

We numerically solve the equations of motion (2) with $\gamma=0$ at two values of $\Delta$ corresponding to chaotic and regular regimes of the center-of-mass motion. In accordance with the Lyapunov map in Fig. 2, behavior of the Bloch component $u$ in Fig. 3, and the simple estimates given above, we expect the chaotic scattering of atoms at $\Delta=0.2$ and their regular motion at $\Delta=1$. Trajectories in the real and momentum spaces for 50 atoms with the same initial momentum, $p_{0}=10$, and initial positions in the range $-\pi / 10 \leq x \leq \pi / 10$ are shown in Figs. 4 and 5 , respectively, at the fixed value of the recoil frequency $\omega_{r}=10^{-3}$. The upper panels in Figs. 4 and 5 illustrate the broad distributions of the atoms in the $x$ and $p$ spaces in the regime of chaotic scattering that contrasts strictly with those obtained in the case of the regular scattering at $\Delta=1$ (Figs. 4b and 5b). In order to create narrow atomic beams, one may use a pair of light masks. The first SW with a red large detuning $(\Delta<0)$ splits the initial atomic beam into a number of narrow beams with the widths much smaller than the optical wavelength which then cross the second SW. The method for creating narrow wave packets in the nonadiabatic regime of scattering has been proposed in Ref. [29].

Dissipative chaotic scattering and simulation of a real experiment. We have illustrated in Figs. 4 and 5 the Hamiltonian chaotic scattering that may occur in the absence of any losses. To simulate trajectories of spontaneously emitting atoms we use the standard stochastic wave-function technique (see, for example, 30 32]) for solving Eqs. (2). The integration time is divided into a large number of small time intervals $\delta \tau$. At the end of the first one $\tau=\tau_{1}$ the probability of spontaneous emission, $s_{1}=\gamma \delta \tau\left|a_{\tau_{1}}\right|^{2} /\left(\left|a_{\tau_{1}}\right|^{2}+\left|b_{\tau_{1}}\right|^{2}\right)$, is computed and compared with a random number $\varepsilon$ from the interval $[0,1]$. If $s_{1}<\varepsilon_{1}$, then one prolongs the integration but renormalizes the state vector in the end of the first interval at $\tau=\tau_{1}^{+}: a_{\tau_{1}^{+}}=a_{\tau_{1}} / \sqrt{\left|a_{\tau_{1}}\right|^{2}+\left|b_{\tau_{1}}\right|^{2}}$ and $b_{\tau_{1}^{+}}=b_{\tau_{1}} / \sqrt{\left|a_{\tau_{1}}\right|^{2}+\left|b_{\tau_{1}}\right|^{2}}$. If $s_{1} \geq \varepsilon_{1}$, then the atom emits a spontaneous photon and performs the jump to the ground state at $\tau=\tau_{1}$ with $A_{\tau_{1}}=\alpha_{\tau_{1}}=\beta_{\tau_{1}}=0$, $B_{\tau_{1}}=1$. Its momentum in the $x$ direction changes for a random number from the interval $[0,1]$ due to the photon recoil effect, and the next time step commences.

We simulate lithium atoms with the relevant transition $2 S_{1 / 2}-2 P_{3 / 2}$, the corresponding wavelength $\lambda_{a}=$ $670.7 \mathrm{~nm}$, recoil frequency $\nu_{\mathrm{rec}}=63 \mathrm{KHz}$, and the decay time $T_{\mathrm{sp}}=2.73 \cdot 10^{-8} \mathrm{~s}$. With the maximal Rabi frequency $\Omega_{0} / 2 \pi \simeq 126 \mathrm{MHz}$ and the radius of the laser beam $r=0.05 \mathrm{~cm}$ one gets $\omega_{r}=10^{-3}, \sigma_{\tau}=400$, and $\gamma=0.05$. Simulated trajectories in the real space for 50 spontaneously emitting atoms under the same conditions as in Fig. 4 are shown in Fig. 6. Even though deterministic Hamiltonian chaos is masked by random events of spontaneous emission, nevertheless the spatial and momentum (not shown) distributions are much broader at those values of $\Delta$ at which the Hamiltonian center-of-mass motion is chaotic. Namely the chaotic Hamiltonian walking is eventually responsible for divergency of atomic beams in the real and momentum spaces.

To simulate a real experiment we consider a beam of $10^{4}$ lithium atoms with the initial Gaussian distribution (the $\mathrm{rms} \sigma_{x}=\sigma_{p}=2$ and the average values, $x_{0}=0$, and momentum, $p_{0}=10$ ) and compute their distribution at a fixed moment of time. In Fig. 7 $\mathrm{a}$ we compare the atomic position distributions at $\tau=1000$ for the chaotic scattering at $\Delta=0.2$ (bold curve) and the regular scattering at $\Delta=1$ (dashed curve) when neglecting spontaneous emission. The difference is evident. In the regime of the chaotic scattering at $\Delta=0.2$ atoms are distributed more or less homogeneously over a large distance of 8 wavelengths along the $x$-axis whereas they form a few peaks in a much more narrow interval under the conditions of 
the regular scattering at $\Delta=1$. Figure 7 p demonstrates the distributions of spontaneously emitting atoms at the normalized decay rate $\gamma=0.05$ under the same conditions as in Fig. 7 a. The regularly scattered atoms at $\Delta=1$ (dashed curve) form the contrast atomic relief with the bifurcated peaks around the first few SW nodes at $x= \pm 1 / 4$, $x= \pm 3 / 4$ and $x=5 / 4$. The distribution of chaotically scattered atoms at $\Delta=0.2$ (bold curve) has the peaks without any bifurcation at $x= \pm 1 / 4$ and $x=3 / 4$ with a smaller number of atoms in each one. Moreover, this distribution is less contrast as compared to the previous one. Thus, we predict that under the conditions of chaotic scattering there should appear less contrast and more broadened atomic reliefs as compared to the case of regular scattering because a large number of atoms are expected to be deposited between the nodes as a result of chaotic walking along the SW axis. The effect is expected to be more prominent under the coherent evolution but it seems to be observable with spontaneously emitting atoms as well.

We predict that experiments on the scattering of atomic beams at a SW laser field can directly image chaotic walking of atoms along the SW. In a real experiment the final spatial distribution can be recorded via fluorescence or absorption imaging on a CCD, commonly used methods in atom optics experiments yielding information on the number of atoms and the cloud's spatial size. The other possibility is a nanofabrication where the atoms after the interaction with the SW are deposited on a silicon substrate in a high vacuum chamber. In this case the spatial distribution can be analyzed with an atomic force microscope. As to the momentum distribution, it can be measured, for example, by a time-of-flight technique. The modern tools of atom optics enable to create narrow initial atomic distributions in position and momentum, reduce coupling to the environment and technical noise, create one-dimensional optical potentials, and to measure spatial and momentum distributions with high sensitivity and accuracy [9].

Conclusion. - We have simulated the new type of atomic diffraction at a SW without any modulation of its parameters and shown that it can be observed in real experiments. That would be the prove of existence of the novel type of atomic motion, chaotic walking in a deterministic environment. The effect could be used in optical nanolithography to fabricate complex atomic structures on substrates.

Acknowledgments. - The work was supported by the Integration grant from the Far-Eastern and Siberian branches of the Russian Academy of Sciences (12-II-0-02001), and by the Program "Fundamental Problems of Nonlinear Dynamics in Mathematics and Physics". I thank L.E. Konkov and M.Yu. Uleysky for the help in preparing some figures.

\section{REFERENCES}

[1] Kazantsev A.P., Ryabenko G.A., Surdutovich G.I. and Yakovlev V.P., Phys. Rep., 129 (1985) 75.

[2] Arimondo E., Bambini A. and Stenholm S., Phys. Rev., 24 (1981) 898.

[3] Adams C.S., Sigel M. and Mlynek J., Phys. Rep., 240 (1994) 143. 2001.

[4] Timp G. et al, Phys. Rev. Lett., 69 (1992) 1636.

[5] McClelland J. J. et al, Science, 262 (1993) 877.

[6] Jürgens D. et al, Phys. Rev. Lett., 93 (2004) 237402.

[7] Graham R., Schlautmann M. and Zoller P., Phys. Rev. A, 45 (1992) R19.

[8] Kolovsky and Korsch H.J., Phys. Rev. A, 57 (1998) 3763.

[9] Steck D.A., Oskay W.H. and Raizen M.G., Science, 293 (2001) 274.

[10] Robinson J.S. et al, Phys. Rev. Lett., 76(1996) 3304.

[11] Chaudhury S. et al, Science, 461 (2009) 768.

[12] Haake F., Quantum signatures of chaos (Springer-Verlag, Berlin) 2001.

[13] Stockmann H. J., Quantum Chaos: An Introduction (Cambridge University Press, Cambridge) 1999.

[14] Kon'kov L.E. and Prants S.V., JETP Letters, 65 (1997) 833.

[15] Prants S.V. and Kon'kov L.E., JETP Letters, 73 (2001) 1801.

[16] Prants S.V., Edelman M. and Zaslavsky G.M., Phys. Rev. E, 66 (2002) 046222

[17] Prants S.V. and Sirotkin V.Yu., Phys. Rev. A, 64 (2001) 033412.

[18] Argonov V.Yu. and Prants S.V., Phys. Rev. A, 75 (2007) 063428.

[19] Prants S.V., JETP Letters, 75 (2002) 651.

[20] Argonov V.Yu. and Prants S.V., JETP, 96 (2003).

[21] Argonov V.Yu. and Prants S.V., Phys. Rev. A., 71 (2005) 053408.

[22] Argonov V.Yu. and Prants S.V., Phys. Rev. A, 78 (2008) 043413.

[23] Petit J.M. and Henon M., Icarus, 60 (1986) 536.

[24] Budyansky M., Uleysky M. and Prants S., Physica D, 195 (2004) 369.

[25] Budyansky M.V., Uleysky M.Yu. and S.V. Prants S.V., JETP, 99 (2004) 1018.

[26] Gaspard P., Chaos, Scattering and Statistical Mechanics, (Cambridge University Press, Cambridge) 1998.

[27] Eckhardt B., Physica D, 33 (1988) 89.

[28] Aguirre J., Viana R.L. and Sanjuan M.A.F., Rev. Mod. Phys., 81 (2009) 333.

[29] Fedorov M.V., Efremov M.V., Yakovlev V.P. and Schleich W.P., JETP, 97 (2003) 522.

[30] Carmichael H.J., An open systems approach to quantum optics (Springer-Verlag, Berlin) 1993.

[31] Dalibard J., Castin Y. and Mölmer K., Phys. Rev. Lett., 68 (1992) 580.

[32] Dum R., Zoller P. and Ritsch H., Phys. Rev. A, 45 (1992) 4879 . 